\title{
Diagnosis of Biological Activities by Mass Spectrometry ${ }^{\dagger}$
}

\author{
Vincent Carré ${ }^{1}$, Pierre Leroy ${ }^{2}$ and Patrick Chaimbault ${ }^{1, *}$ \\ 1 Université de Lorraine, LCP-A2MC, 1, Boulevard Arago, F-57078 Metz, France; \\ vincent.carré@univ-lorraine.fr \\ 2 Université de Lorraine, CITHEFOR, F-54000 Nancy, France; pierre.leroy@univ-lorraine.fr \\ * Correspondence: patrick.chaimbault@univ-lorraine.fr; Tel.: +33-372-74-91-88 \\ + Presented at Natural Products and the Hallmarks of Chronic Diseases-COST Action 16112, Luxemburg, \\ 25-27 March 2019.
}

Published: 29 April 2019

\begin{abstract}
Oxidative reactions are vital but also cause important stresses and cellular damages resulting in cancers, cardiovascular or neurodegenerative diseases. Antioxidant secondary metabolites from plant can be mobilized for the cell defense and their main source is precisely the food intake such as vegetables, fruits or beverages. Screening natural active metabolites in plants requires different analytical techniques among which mass spectrometry became one of the most popular, not just because of its ability to provide structural information on involved molecules but also because this technique belongs to the arsenal of diagnostic tool for the determination of biological activities.
\end{abstract}

Keywords: mass spectrometry; imaging mass spectrometry; plants; anti-cancer; host-pathogen relation ship

\section{Introduction}

Oxygen is vital for life however; the resulting oxidative reactions may also be the cause of cellular stresses degenerating into cancers, cardiovascular or neurodegenerative diseases. By chance, the plant world provides many antioxidant secondary metabolites, which can be mobilized for the cell defense. Most of the time, the source of this "natural protection" is coming from the dietary intake.

The identification of natural active compounds is of the greatest importance. They can be purified from plants using many different techniques (specific extraction, chromatography) and most of the time their structural identification also requires several analytical methods. All these steps often require long optimization.

In the last 25 years, mass spectrometry (MS) became one of the most useful technique for structural elucidation of molecules of biological interest. Furthermore, MS also became a powerful tool for the direct diagnosis of their biological activities. Indeed, Mass Spectrometry is not just appreciable because of its ability to provide structural information on molecules; this technique also belongs to the arsenal of tools able to monitor the disturbances occurring in the cell cycles, able to fish active metabolites in plants or even to highlight biomarkers in tissues directly.

\section{Monitoring and Evaluation of Disturbances Occurring in the Cell Cycles and Pathophysiological Events}

The measurement of very small messenger (molecular weight $<50 \mathrm{u}$ ) such as nitric oxide (NO) is particularly difficult in Mass Spectrometry. 
NO plays a pivotal role in vascular tone homeostasis and inflammation. During aging and in case of cardiovascular diseases (CVD), endogenous NO production decreases. Its origin includes both endogenous and exogenous sources (drinking water and food intake). Oral administration of Snitrosothiols (RSNOs) appears promising to restore the NO pool in CVD and to fight against oxidative stress and inflammation.

In the presented example, intestine permeability of RSNO and especially S-nitrosoglutathione (GSNO) are studied by using in vitro models by measuring metabolites (nitrate, nitrite ions, and lowand high-molecular mass RSNOs). An analytical method relying upon liquid chromatographyelectrospray ionization-tandem mass spectrometry (LC-ESI-MS/MS), and able to distinguish between the exogenous origin of NO species and other sources (endogenous synthesis and diet) was developed [1]. RSNO were labeled with the stable nitrogen isotope ${ }^{15} \mathrm{~N}$ (exhibiting a very low natural abundance compared to ${ }^{14} \mathrm{~N}$ ). 2,3-Diaminonaphthalene (DAN) assay was selected for nitrite derivatization and the resulting product, i.e., 2,3-naphthotriazole (NAT), was examined in LCMS/MS conditions. An interference was observed between unlabeled NAT and ${ }^{15} \mathrm{~N}$ labeled one, due to the presence of ${ }^{13} \mathrm{C}$ (stable natural isotope abundance of $1 \%$ ), and the interference ratio was about $3 \%$. Besides, an original new mass fragmentation pattern offering high sensitivity was optimized by using appropriate mobile phase containing acetonitrile and detection mode with collision the HCD cell (Higher-energy Collisional Dissociation HCD).

The present analytical method was validated and applied to GSNO permeability studies. The different NO species were measured in apical/basolateral, donor-acceptor compartments of the models. The results situate this drug candidate within the middle permeability class.

\section{Fishing Active Metabolites from Plant Extracts}

Plants are source of natural active compounds for a long time. They are involved in many fields of application such as medicine or cosmetic. The search for new natural active molecules against cancer (and obviously other diseases) in plant extracts can be directly obtained through the analysis their extract by Matrix Assisted Laser Desorption/Ionization-Time of Flight Mass Spectrometry (MALDI-TOFMS) or Fourier Transform Ion Cycloton Resonance Mass Spectrometry (MALDIFTICRMS). As described in Figure 1, the first step requires their contact with implied target proteins. The target protein acts as a filter, which retain the candidate molecules. The complex protein-ligand is analyzed by Mass Spectrometry and the direct detection of the ligand reflects its potential inhibition activity against the target. Screening tests have already been developed for the ligands of tubulin $[2,3]$, dihydroxyfolate reductase (DHFR) [4] and recently for reversible and irreversible ligands of CDC25 phosphatases (isoforms A et C) [5]. This approach demonstrates its effectiveness with Madagascar periwinkle for tubulin, with Colchicum and green tea for DHFR and some promising plants are currently evaluated on CDC25s.

\section{Highlighting Biomarkers in Tissues}

The host-pathogen relationship between plant and their pathogens can also directly studied by Mass spectrometry. Throughout the year, pathogens infest grapevine causing severe culture damages. Understanding host-pathogen relationships and finding the source of the resistance observed with some vine strains may be the solution to avoid heavy phytosanitary treatments.

The direct infusion of extracts of healthy and infected leaf in electrospray-Fourier transform ion cyclotron resonance mass spectrometry (ESI-FTICRMS) provides a straightforward information on grapevine defense against downy mildew (Plasmopara viticola) [6]. The comparison of the MS profiles highlights several classes of involved secondary metabolites (disaccharides, acyl lipids and sulfoquinovosyl diacylglycerols) which were identified thanks to high resolution MS and tandem MS (Figure 2) (MS/MS). Following the selection of 19 marker ions, Principal Component Analyses (PCA) show a clear difference between infected and healthy samples highlighting that the corresponding metabolites are likely involved in the plant defense. 


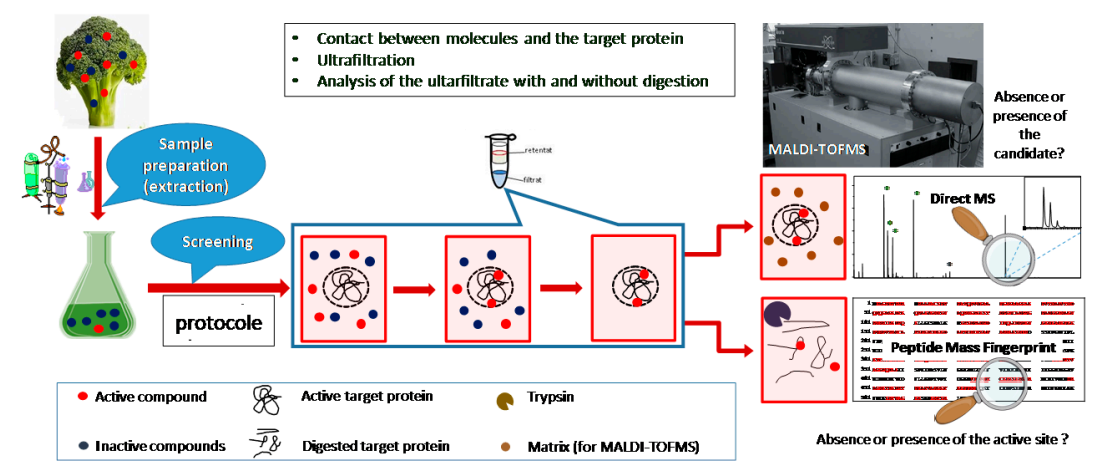

Figure 1. Principle of the direct screening protocol. The plant is first undergoing extraction with appropriate solvents. The, the extract is put in contact with the target protein to form a complex with the active candidates. The complex is ultra-filtrated to eliminate non active compounds before MALDI-TOFMS analysis. The retentate is directly submitted to MS analysis to check if one candidate (or even several) bound to the target protein and after a protein digestion to check the presence of the target active site by peptide mass fingerprint (PMF), it can be determined if the inhibition is reversible or not.

These results can be interestingly completed by Imaging Mass Spectrometry (IMS), which allows the direct spatial localization of metabolites of interest (stilbenes [7], flavonoids [8] or ellagic acid) in the leaf tissues.

When necessary, LC-MS and LC-MS/MS can be used to complete the information, especially regarding the quantification of specific metabolites of interest in leaf extracts.

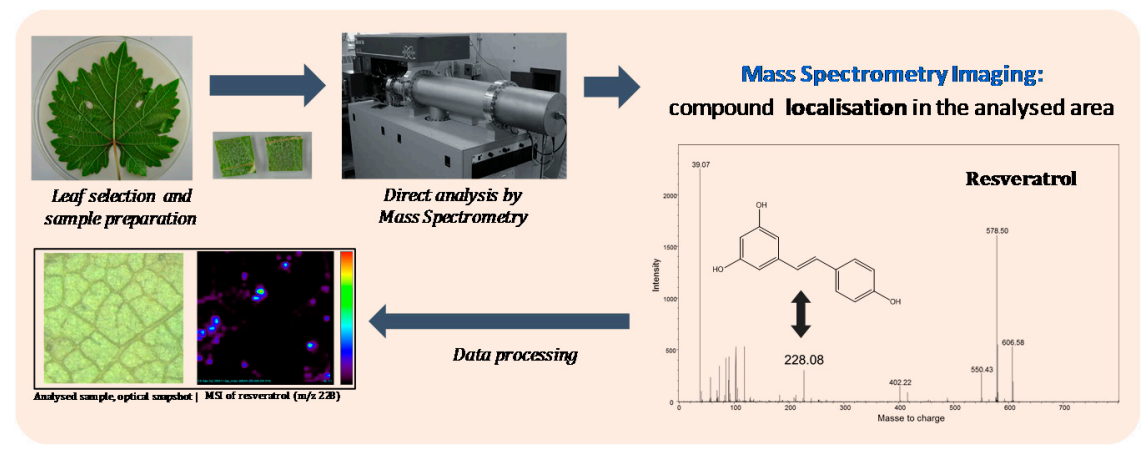

Figure 2. General scheme of Imaging Mass Spectrometry by (MA)LDI-TOFMS. The sample, here an infected vine leaf (with Plasmopara viticola), is cut and fixed to the MALDI plate with or without matrix deposition. The MALDI plate is introduced in the mass spectrometer. A grid pattern covering the surface to be analyzed is defined. The sample is then mapped; each pixel of the grid is composed of mass spectra. Thank to data processing software, the image of the distribution of each ion of interest can be build (here m/z 228 for resveratrol). The image can be compared with control sample or superposed to the initial photo.

Author Contributions: V.C., P.L. and P.C. performed the research, analyzed the results and drafted the article.

Funding: This article is based upon work from COST Action NutRedOx-CA16112 supported by COST (European Cooperation in Science and Technology).

Conflicts of Interest: The authors declare no conflict of interest.

\section{References}

1. Yu, H.; Bonetti, J.; Gaucher, C.; Fries, I.; Vernex-Loset, L.; Leroy, P.; Chaimbault, P. Higher-energy Collision Dissociation for the quantification by liquid chromatography-tandem ion trap mass spectrometry of nitric oxide metabolites coming from S-nitrosoglutathione in an in vitro model of intestinal barrier. Rapid Commun. Mass Spectrom. 2019, 33, 1-11, doi:10.1002/rcm.8287. 
2. Hannewald, P.; Maunit, B.; Muller, J.-F. Tubulin-binding drug screening by MALDI-TOFMS. Anal. Chem. 2006, 78, 4390-4397, doi:10.1021/ac0601462.

3. Efe, E.Y.; Mazumder, A.; Lee, J.-Y.; Gaigneaux, A.; Radogna, F.; Nasim, M.J.; Christov, C.; Jacob, C.; Kim, K.-W.; Dicato, M.; et al. Tubulin-binding anticancer polysulfides induce cell death via mitotic arrest and autophagic interference in colorectal cancer. Cancer Lett. 2017, 410, 139-157, doi:10.1016/j.canlet.2017.09.011.

4. Hannewald, P.; Maunit, B.; Muller J.-F. Screening of DHFR-binding drugs by MALDI-TOFMS. Anal. Bioanal. Chem. 2008, 392, 1335-1344, doi:10.1007/s00216-008-2409-x.

5. Sibille, E.; Bana, E.; Chaouni, W.; Diederich, M.; Bagrel, D.; Chaimbault, P. Development of a MALDI-MS screening test to evidence reversible and irreversible inhibitors of CDC25 phosphatases. Anal. Biochem. 2012, 430, 83-91, doi:10.1016/j.ab.2012.08.006.

6. Becker, L.; Poutaraud, A.; Hamm, G.; Muller, J.-F.; Merdinoglu, D.; Carré, V.; Chaimbault, P. Metabolic study of grapevine leaves infected by downy mildew using negative ion electrospray-Fourier transform ion cyclotron resonance mass spectrometry. Anal. Chim. Acta 2013, 79, 544-551, doi:10.1016/j.aca.2013.07.068.

7. Becker, L.; Carré, V.; Poutaraud, A.; Merdinoglu, D.; Chaimbault, P. MALDI mass spectrometry imaging for the simultaneous location of resveratrol, pterostilbene and viniferins on grapevine leaves. Molecules 2014, 19, 10587-10600, doi:10.3390/molecules190710587.

8. Becker, L.; Bellow, S.; Carré, V.; Latouche, G.; Poutaraud, A.; Merdinoglu, D.; Brown, S.; Cerovic, Z.; Chaimbault, P. Correlative analysis of fluorescent phytoalexins by mass spectrometry imaging and fluorescence microscopy in grapevine leaves. Anal. Chem. 2017, 89, 7099-7106, doi:10.1021/acs.analchem.7b01002. 